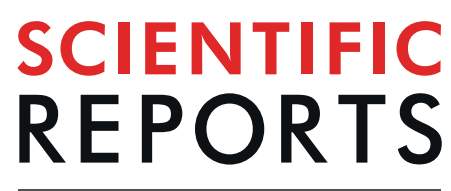

\title{
mTORC1 activity is essential for erythropoiesis and B cell lineage commitment
}

\begin{abstract}
Natasha Malik $\mathbb{D}^{1}$, Karen M. Dunn $\mathbb{D}^{1}{ }^{1}$, Jennifer Cassels ${ }^{1}$, Jodie Hay ${ }^{1}$, Christopher Estell ${ }^{1}$, Owen J. Sansom $\mathbb{D i D}^{1,2}$ \& Alison M. Michie $\mathbb{D}^{1 *}$

Mechanistic target of rapamycin (mTOR) is a serine/threonine protein kinase that mediates phosphoinositide-3-kinase (PI3K)/AKT signalling. This pathway is involved in a plethora of cellular functions including protein and lipid synthesis, cell migration, cell proliferation and apoptosis. In this study, we proposed to delineate the role of mTORC1 in haemopoietic lineage commitment using knock out (KO) mouse and cell line models. Mx1-cre and Vav-cre expression systems were used to specifically target Raptor ${ }^{f / f l}$ (mTORC1), either in all tissues upon poly(I:C) inoculation, or specifically in haemopoietic stem cells, respectively. Assessment of the role of mTORC1 during the early stages of development in $\mathrm{Vav}$-cre ${ }^{+}$Raptor $^{f l f l}$ mice, revealed that these mice do not survive post birth due to aberrations in erythropoiesis resulting from an arrest in development at the megakaryocyteerythrocyte progenitor stage. Furthermore, Raptor-deficient mice exhibited a block in B cell lineage commitment. The essential role of Raptor (mTORC1) in erythrocyte and B lineage commitment was confirmed in adult $M \times 1-\mathrm{cre}^{+}$Raptor $^{f l / f l}$ mice upon cre-recombinase induction. These studies were supported by results showing that the expression of key lineage commitment regulators, GATA1, GATA2 and PAX5 were dysregulated in the absence of mTORC1-mediated signals. The regulatory role of $\mathrm{mTOR}$ during erythropoiesis was confirmed in vitro by demonstrating a reduction of $\mathrm{K} 562$ cell differentiation towards RBCs in the presence of established mTOR inhibitors. While mTORC1 plays a fundamental role in promoting RBC development, we showed that $\mathrm{mTORC2}$ has an opposing role, as Rictor-deficient progenitor cells exhibited an elevation in RBC colony formation ex vivo. Collectively, our data demonstrate a critical role played by $\mathrm{mTORC1}$ in regulating the haemopoietic cell lineage commitment.
\end{abstract}

Recent studies of erythroid differentiation have revealed differences in the lineage potential of distinct cellular subsets between fetal and adult progenitor populations, with a higher proportion of megakaryocyte-erythroid progenitors arising from multipotent and oligopotent progenitor subsets during fetal haemopoiesis, while adult erythroid-committed cells are derived predominantly from unipotent progenitors ${ }^{1}$. These studies suggest that the erythroid cell lineage may be differentially regulated during ontogeny. Multipotent progenitor (MPP) cells give rise to lymphoid-primed multipotent progenitors (LMPPs) and oligopotent common myeloid progenitors (CMPs). LMPPs have the capacity to differentiate towards common lymphoid progenitors (CLPs) mainly, with reduced megakaryocyte-erythroid progenitor (MEP) potential, while CMPs branch towards MEPs and granulocyte-macrophage progenitor (GMP) cells ${ }^{2}$. MEPs further commit to the erythroid lineage through formation of burst-forming unit-erythroid (BFU-E) and then colony-forming unit-erythroid (CFU-E) cells, which then progress through erythroblast stages, reticulocyte maturation and terminal differentiation into red blood cells (RBCs) $)^{3}$.

Erythropoiesis is governed by the complex regulation of key transcription factors (TFs), enabling a balance between self-renewal and differentiation of erythrocyte progenitors and terminal erythroblasts. The zinc-finger TFs GATA1 and GATA2 play critical and non-redundant roles during erythroid maturation. GATA2 is expressed in HSCs and early progenitor populations regulating the expression of self-renewal genes, and genes responsible for initiating GATA1 expression. GATA1 plays a vital role in erythroid differentiation, sustaining its own expression and suppressing GATA2 expression, a process called GATA factor switching. GATA1-deficient $\left(G A T A 1^{-I-}\right)$ 


\begin{tabular}{|c|c|c|c|c|c|c|c|}
\hline Age & $\begin{array}{l}\text { Medelian } \\
\text { Ratio }\end{array}$ & $\mathrm{Cre}^{-}$Raptor $^{\mathrm{w} t / \mathrm{fl}}$ & $\begin{array}{l}\text { Cre }^{-} \\
\text {Raptor }^{\mathrm{f} / \mathrm{fl}}\end{array}$ & $\begin{array}{l}\mathrm{Cre}^{+} \\
\text {Raptorwt/fl }^{\text {wh }}\end{array}$ & $\begin{array}{l}\text { Cre }^{+} \\
\text {Raptor }^{\mathrm{f} / \mathrm{fl}}\end{array}$ & \begin{tabular}{|l|l|} 
Chi $^{2}$ \\
Value
\end{tabular} & $p$ Value \\
\hline \multirow{2}{*}{ Weaning } & Expected & 6.5 & 6.5 & 6.5 & 6.5 & 14.92 & $* *$ \\
\hline & Actual & 13 & 9 & 4 & 0 & & \\
\hline \multirow{2}{*}{ E13 } & Expected & 12.25 & 12.25 & 12.25 & 12.25 & 2.92 & 0.50 \\
\hline & Actual & 12 & 8 & 15 & 14 & & \\
\hline
\end{tabular}

Table 1. Deletion of Raptor in the haemopoietic lineages leads to death prior to weaning. The expected mendelian ratios and actual genotyped ratios of $\mathrm{Vav}_{\text {- } \mathrm{Cre}^{+/-}} \mathrm{Raptor}^{\mathrm{tt} / f l}$ mouse matings are shown at weaning $(4 \mathrm{wk} ; \mathrm{n}=26)$ and at embryonic day $13(\mathrm{E} 13 ; \mathrm{n}=49)$. Chi test values and statistical analyses are shown $(* * \mathrm{p} \leq 0.001)$. $\mathrm{p}$ value calculated by the Chi Test which determines $\mathrm{p}$ value based on degrees of freedom.

murine embryos give rise to lymphoid cells and non-haemopoietic tissues, but not a mature erythroid population, resulting from a block at the pro-erythroblast stage due to increased apoptosis ${ }^{4}$. Ikaros also plays a role in fetal and adult erythropoiesis and erythroid lineage commitment, with Ikaros gene silencing leading to an irreversible switch to the myeloid lineage ${ }^{5}$. PU.1 TF is an established master regulator in haemopoiesis involved in primitive cell fate decisions. PU.1 expression levels determine myeloid and lymphoid cell fates: higher expression of PU.1 leads to myeloid cell fate while a lower expression to a lymphoid fate ${ }^{6}$. The myeloid cell fate is not solely regulated by PU.1 expression, but also the inhibition of $G A T A 1^{7}$ and the expression of $C E B P \alpha^{8}$. PU.1 and GATA1 physically interact to regulate lineage fate where upregulation of GATA1 inhibits $P U .1$ transcription and promotes erythroid lineage differentiation ${ }^{9}$, while expression of PU.1 inhibits GATA1 expression promoting myeloid lineage fate ${ }^{10}$. GATA 1 binds to the promoter region of erythroid-Krüppel-like factor $(e-K L F / K l f 1)^{11}$ which is vital for erythropoiesis, regulating MEP lineage fate decisions during erythrocyte development and globin switching from $\gamma$ - to $\beta$-GLOBIN during erythrocyte maturation ${ }^{12}$. In humans, $\beta$-GLOBIN is expressed when erythropoiesis moves to the bone marrow (BM). Initially, the yolk-sac expresses $\varepsilon-G L O B I N$, followed by the expression of $\gamma-G L O B I N$ in the fetal liver (FL) and spleen. Therefore, $e-K L F$ mediated $\beta$-GLOBIN expression is vital for mature erythrocyte development ${ }^{13}$. While $e-K L F$ plays a role in erythropoiesis, $K L F 2$ is involved in endothelial growth, vascular remodeling and inflammation responses ${ }^{14}$, which is vital for embryonic development. Lower expression levels of $P U .1$ skew progenitors towards a lymphoid lineage enhancing $E 2 A$ expression, a TF involved at the earliest stages of $\mathrm{B}$ cell development. Lack of $E 2 A$ leads to a block in B cell development at the pre-proB and pro-B stages ${ }^{15}$. E2A drives the expression of early B cell factor 1 ( $E B F 1$ ), which together with $E 2 A$, regulate $P A X 5$ and $R A G$ genes responsible for $\mathrm{B}$ cell lineage commitment and $\mathrm{V}(\mathrm{D}) \mathrm{J}$ recombination to form the pre-B cell receptor complex on pre-B cells ${ }^{16}$.

The mTOR/AKT signaling pathway has been shown to play a vital role in haemopoietic lineage development and maturation. The mTOR pathway is activated by a variety of growth factor receptors and nutrients including glucose, iron and amino acids. mTOR forms two different complexes - mTORC1 and mTORC2. mTORC1 is composed of 6 proteins and mTORC 2 of 7 proteins. Of these, mTOR kinase is common, along with GBL, DEPTOR, and the TTI1/TEL2 complex. The subunits that make the respective complexes unique are RAPTOR (rapamycin TOR-sensitive) and PRAS40 for mTORC1 and RICTOR (rapamycin TOR-insensitive), mSIN1, and PROTOR1/2 for mTORC2. AKT lies upstream of mTORC1 and downstream of mTORC2 thus playing a crucial role in mTOR pathway regulation ${ }^{17}$. Downstream of mTORC1, S6 kinase 1 (S6K1) inhibits mTORC2 activity, thereby creating a negative feedback loop and another regulatory mechanism for this pathway ${ }^{18}$.

A critical role of mTORC1 has been identified in erythropoiesis whereby mTORC1 is regulated by dietary iron and Raptor ablation at the haemopoietic stem cell (HSC) stage leads to perinatal lethality ${ }^{19}$. Furthermore Raptor $\mathrm{KO}$ and overexpression leads to microcytic and macrocytic anaemia respectively ${ }^{19}$. However, discrepancies in the field remain, as there is research demonstrating that $\mathrm{mTORC} 1$ inhibition does not cause anaemia ${ }^{20}$ and that mTORC1 inhibition improves anaemia in a sickle cell disease model ${ }^{21}$. In this study, we address the role of Raptor (mTORC1) in normal haemopoietic lineage commitment both in fetal and adult developmental stages, using the cre-LoxP system to excise Raptor (mTORC1) in adult mice (Mx1-cre) and at the HSC stage (Vav-cre) in embryos, as well as the K562 cell line, to address the role of mTOR-mediated signals during RBC differentiation.

\section{Results}

mTORC1 plays a critical role in B cell and RBC lineage development in vivo. Genotyping analyses of $V a v$-cre ${ }^{+l-}$ x Raptor ${ }^{w t / f l}$ breedings revealed that the mice exhibiting the Vav-Raptor KO (Vav-cre ${ }^{-l+}$ Raptor ${ }^{f l}$ ${ }^{f l}$ ) did not reach weaning age $(4 \mathrm{wk})$, while being present at normal ratios during gestation, suggesting that Vav$\mathrm{cre}^{-l+}$ Raptor ${ }^{f l f l}$ mice died perinatally (Table 1). Analysis of embryos (E13-17) revealed a significant downregulation of Raptor expression in FL isolated from Vav-Raptor KO mice as expected, and the embryos were pale during gestation compared to Vav-cre ${ }^{-l-}$ Raptorfl/fl (Vav-Raptor control) (Fig. 1A,B, \& data not shown). Induction of Raptor excision with poly(I:C) in adult Mx1-Raptor cKO mice revealed a significant downregulation in Raptor expression in the BM and spleen (Fig. 1C) together with an increase in splenic weight and cellularity, and BM cellularity in $M x 1$-cre ${ }^{+}$Raptor ${ }^{f l / f l}$ mice (Mx1-Raptor $\mathrm{cKO}$ ) compared to Mx1-cre ${ }^{-}$Raptor ${ }^{\text {fllfl }}$ mice (Mx1-Raptor control) (Fig. 1D-G).

Analysis for the presence of selected haemopoietic lineages in these mouse models revealed a small but significant decrease in the percentage of Ter $119^{+}$erythroid populations in Vav-Raptor KO FL at E15 compared to Vav-Raptor control (Fig. 2A \& Suppl Fig. 1A). Additionally, there was a trend in decrease in the percentage of Ter $119^{+}$erythroid populations in the BM, coupled with a significant increase in percentage of Ter $119^{+}$ 
A

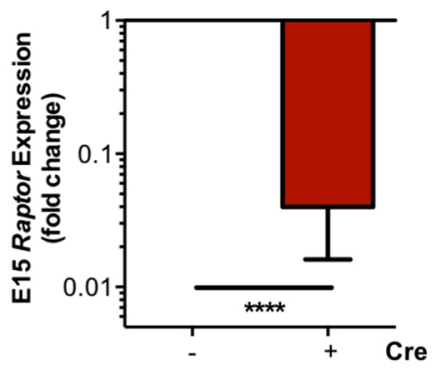

C

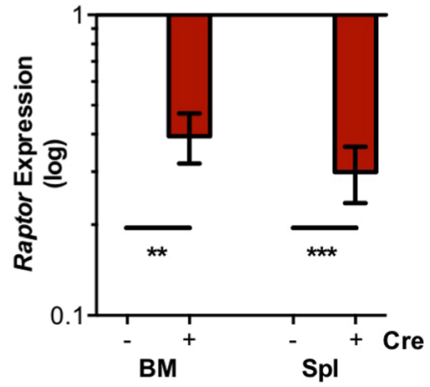

B

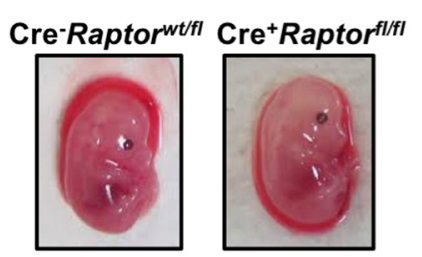

D

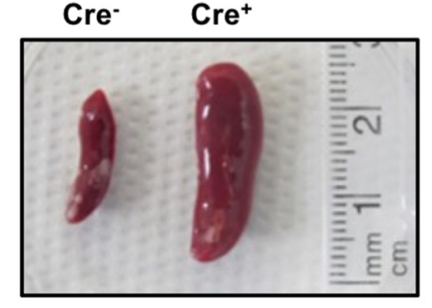

E

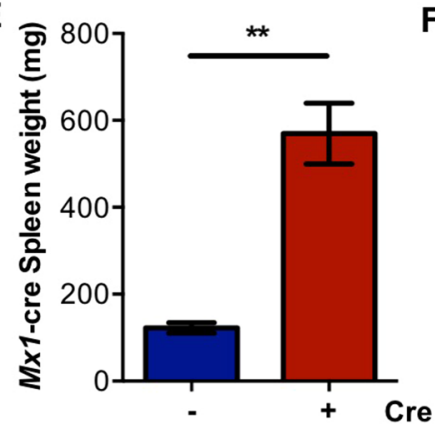

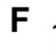

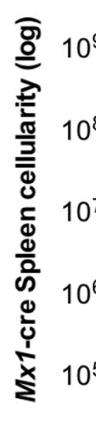

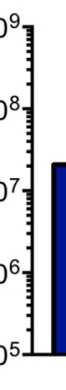

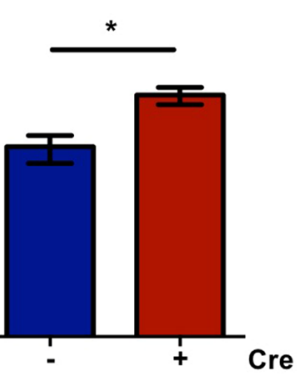

G

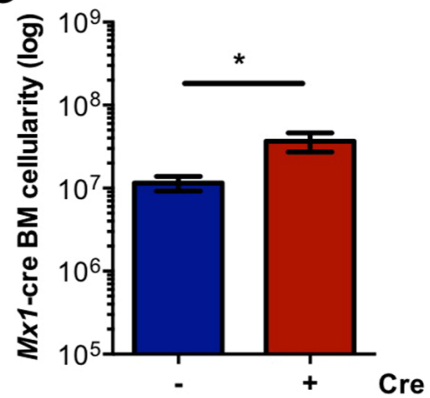

Figure 1. $V a v-$ cre $^{+}$Raptor ${ }^{l l f l}$ mice die perinatally in the absence of raptor expression. (A) Gene expression data showing expression of Raptor with cre expression in Vav-cre ${ }^{+}$Raptor fl/fl (Vav-Raptor KO) compared to $V a v$-cre ${ }^{-}$Raptor fl/fl (control) E15 FL $(\mathrm{n}=6)$. (B) Picture showing the difference in pallor in E13 FL between $V a v$-cre ${ }^{-}$Raptor ${ }^{\mathrm{wt} / \mathrm{fl}}$ and Vav-KO fetal mice; C. Gene expression of Raptor in the BM and spleen in Mx1-cre ${ }^{-}$and $M x 1$-cre ${ }^{+}$Raptor ${ }^{f l / f l}$ mice (red, Mx1-Raptor $\mathrm{cKO} ; \mathrm{n}=5$ ); D. Picture showing splenomegaly in Mx1-Raptor $\mathrm{cKO}$ mice compared to Mx1-Raptor control; Graphs showing spleen weight (mg) (E), spleen cellularity (F) and BM cellularity (G) of $M x 1$-Raptor control (blue) and Mx1-Raptor $\mathrm{cKO}$ mice ( $\mathrm{n} \geq 4)$. All $M x 1$-cre mice were given 4 doses of poly(I:C) and assessed $5 \mathrm{wk}$ post inoculation. Data are expressed as mean \pm SEM, t-test (unpaired) (p $* \leq 0.05 ; * * \leq 0.001 ; * * * 0.0001 ; * * * * \leq 0.00001$.

population in the spleen likely due to increased extramedullary haemopoiesis in Mx1-Raptor cKO mice compared to Mx1-Raptor control (Fig. 2B \& Suppl Fig. 1B). Flow cytometric analysis of B lineage cells revealed a significant downregulation in the percentage of $\mathrm{CD}_{19^{+}} \mathrm{B}$ lineage cells in E17 Vav-KO FL and Mx1-cKO BM, spleen and blood (Fig. 2C-E; Suppl. Fig. 1), coupled with a significant decrease in the number of $\mathrm{CD} 19^{+} \mathrm{B}$ lineage cells in the Mx1-cKO BM (Fig. 2F). These data suggest a vital role for mTORC1 in B cell development. Both the KO models showed a concomitant increase in the percentage of $\mathrm{CD}_{1} \mathrm{~b}^{+} \mathrm{Grl}^{-}$immature myeloid cells, together with a significant decrease in the percentage of $\mathrm{CD} 11 \mathrm{~b}^{+} \mathrm{Gr} 1^{+}$mature myeloid cells (Fig. 2G,H,J; Suppl. Fig. 1). These data were coupled with a significant increase in the number of $\mathrm{CD}_{11 b^{+}} \mathrm{Gr}^{-}$myeloid cells in the Mx1-cKO BM (Fig. 2I), while the mature myeloid cell numbers were unaltered in the $M x 1$-cKO BM and spleen (Fig. $2 \mathrm{~K}$ ). These data suggest that myeloid lineage maturation is deregulated in the absence of Raptor expression. As it is well established that mice can survive in the absence of white blood cell population ${ }^{22}$, the absence of adult Vav-Raptor KO mice is likely due to the critical role of mTORC1 in erythropoiesis which results in the decrease in RBC populations and the pallor of the mice.

mTORC1 plays a critical role in B and RBC lineage commitment in vivo. To gain a deeper understanding of the stage at which Raptor-deficiency blocks lineage commitment/development we carried out flow cytometric analysis on the haemopoietic progenitor populations. Supporting the lack of $\mathrm{CD} 19^{+} \mathrm{B}$ cells, a significant reduction in the percentage of pre-proB and pro-B cells was noted in FL and BM isolated from Vav-Raptor $\mathrm{KO}$ and Mx1-Raptor cKO mice respectively, compared with control mice (Fig. 3A,B; Suppl. Fig. 2). This finding 

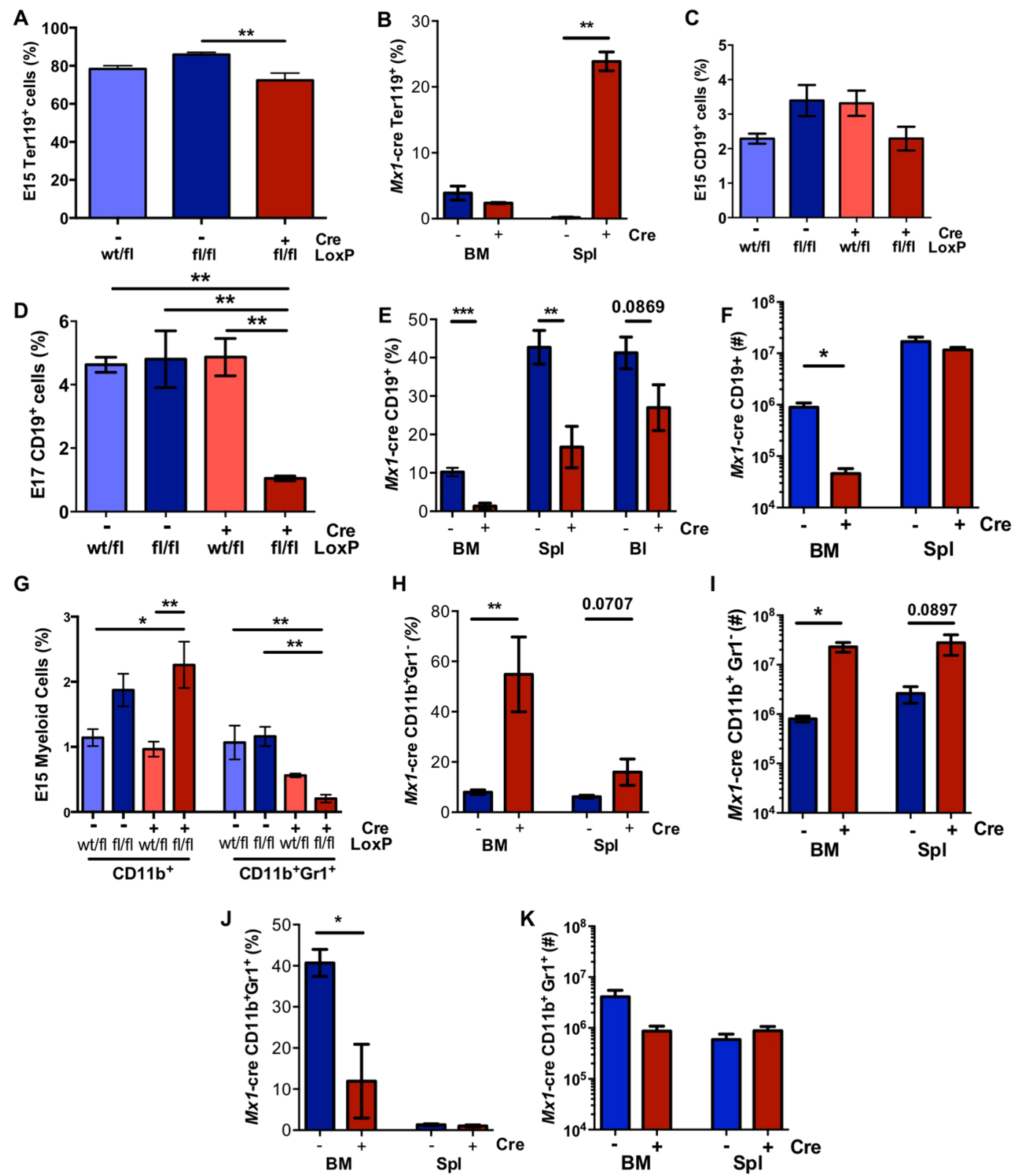

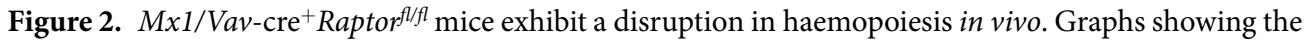
percentage of haemopoietic lineage calculated from flow cytometry data gated as indicated in Supplemental Fig. 1. Average percentage of Ter $119^{+}$erythroid population in Vav-cre ${ }^{-}$Raptor ${ }^{w t / f l}$, Vav-Raptor control, and Vav-Raptor KO E15 FLs ( $\mathrm{n}=7)(\mathrm{A})$ and BM and spleen of Mx1-Raptor control and Mx1-Raptor cKO mice $(\mathrm{n}=3)(\mathrm{B})$; Average percentage of $\mathrm{CD} 19^{+}$B cells in E15 $(\mathrm{n}=6)(\mathrm{C})$ and E17 $(\mathrm{n}=4)(\mathrm{D})$ FLs and BM, spleen and blood of Mx1-Raptor control and Mx1-Raptor $\mathrm{CKO}$ mice $(\mathrm{n}=5)(\mathrm{E})$; Average number of $\mathrm{CD} 19^{+} \mathrm{B}$ cells in $\mathrm{BM}$ and spleen of Mx1-Raptor control and Mx1-Raptor $\mathrm{CKO}$ mice $(\mathrm{n}=5)(\mathbf{F})$; Average percentage of CD11b ${ }^{+} \mathrm{Gr} 1^{-}$ (immature myeloid) and $\mathrm{CD} 11 \mathrm{~b}^{+} \mathrm{Gr}-1^{+}$mature myeloid populations in $V a v$-control, and $V a v$-KO E15 FLs $(\mathrm{n}=6)(\mathrm{G})$, and $\mathrm{CD} 11 \mathrm{~b}^{+} \mathrm{Gr} 1^{-}$(immature myeloid) $(\mathrm{H})$ and $\mathrm{CD} 11 \mathrm{~b}^{+} \mathrm{Gr}-1^{+}$mature myeloid populations $(\mathrm{J})$ in Mx1-Raptor control and Mx1-Raptor $\mathrm{cKO} B M$ and spleens $(\mathrm{n}=5)$. Average number of $\mathrm{CD}_{11 \mathrm{~b}^{+} \mathrm{Gr}^{-}(\mathrm{I})}$ and $\mathrm{CD} 11 \mathrm{~b}^{+} \mathrm{Gr}-1^{+}$myeloid populations (K) in Mx1-Raptor control and Mx1-Raptor cKO BM and spleens $(\mathrm{n}=5)$. All Mx1-cre mice were given 4 doses of poly(I:C) and assessed $5 \mathrm{wk}$ post inoculation. Light blue bars cre $^{-}$Raptor $^{t t / f l}$; blue - cre $^{-}$Raptor ${ }^{f l f l}$; red bars - cre $^{+}$Raptor $^{t t / f l}$; maroon bars - cre ${ }^{+}$Raptor ${ }^{f l f l}$ as indicated. Data are expressed as mean \pm SEM, one way ANOVA $(\mathrm{p} * \leq 0.05 ; * * \leq .001 ; * * * \leq 0.0001 ; * * * * \leq 0.00001)$.

was coupled with a significant elevation in the $\mathrm{Lin}^{-} \mathrm{Sca}-1^{+} \mathrm{CD} 117^{\text {hi }}$ (LSK) population, suggesting a block in B cell development prior to lineage commitment. Of note, no significant differences in LSK and progenitor B cell populations were observed between $\operatorname{VavCr}^{+/-} \mathrm{Raptor}^{w t / w t}$ and $\mathrm{VavCre}{ }^{-}$Raptor ${ }^{l / f l}$ mice, indicating that the phenotypic changes were due to the excision of Raptor (Fig. 3A). Analysis of the proportion of myeloid progenitors in E15 
A
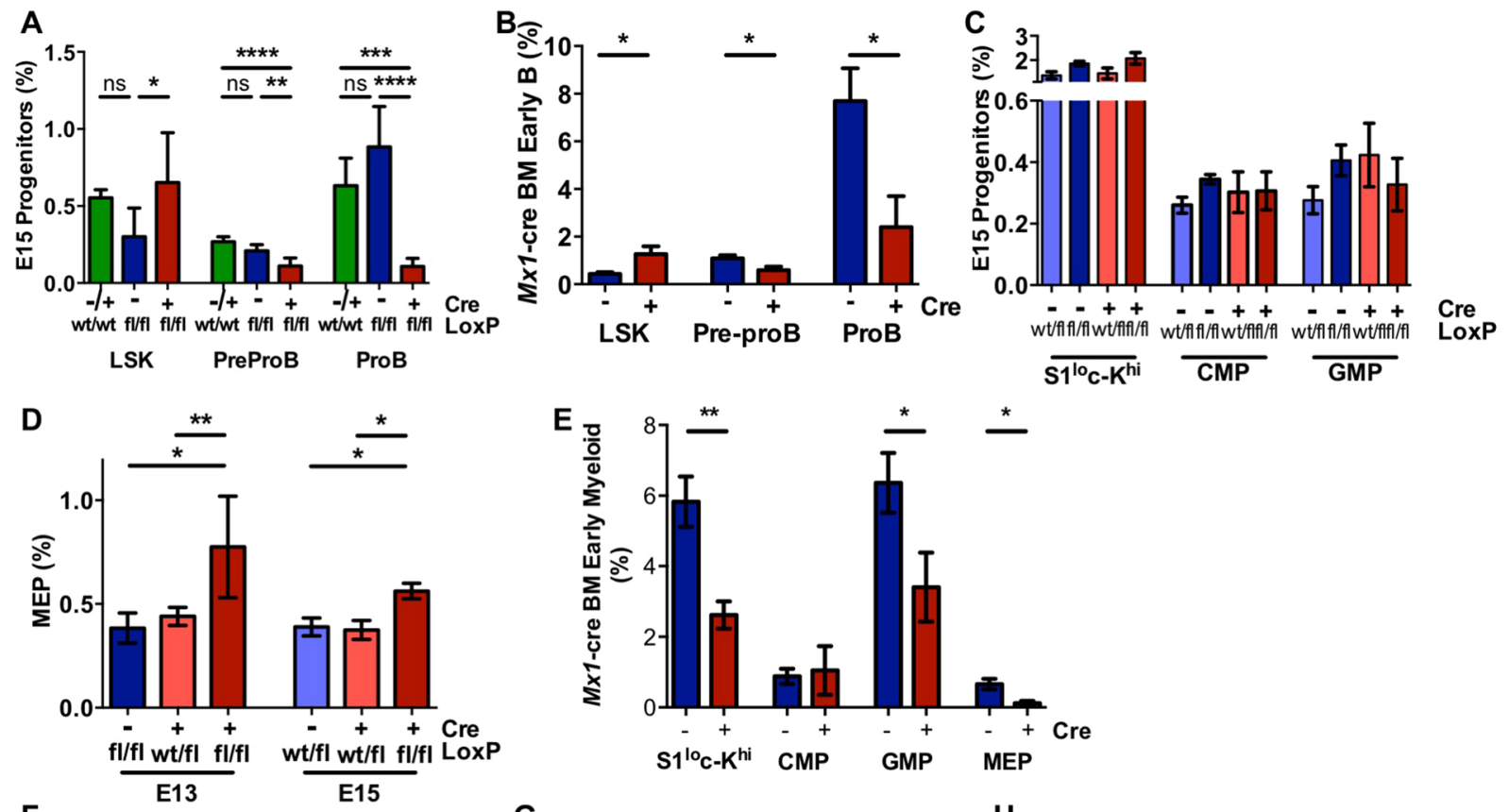

$\mathbf{F}$

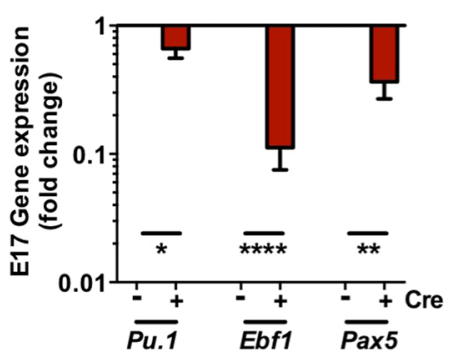

G

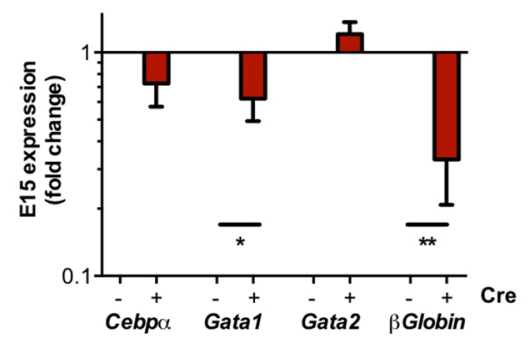

H

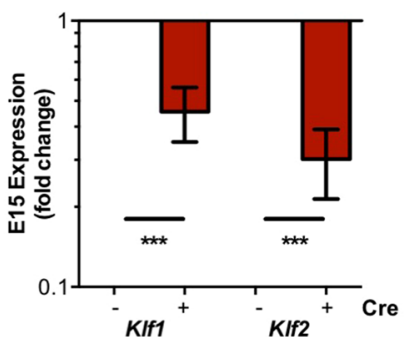

Figure 3. Mxl and $\mathrm{Vav}_{-\mathrm{cre}}^{+}$Raptor ${ }^{\text {fl/fl }}$ mice exhibit a block in B and RBC lineage commitment at the LSK and MEP stage in vivo. Surface expression of $\mathrm{Lin}^{-} \mathrm{Sca}-1^{+} \mathrm{CD} 117^{+}(\mathrm{LSK})$, pre-proB $\left(\mathrm{CD} 117^{+} \mathrm{B} 220^{+} \mathrm{CD} 19^{-}\right)$and proB $\left(\mathrm{CD} 117^{+} \mathrm{CD} 19^{+} \mathrm{B} 220^{+}\right)$populations in E15 Vav-Raptor KO FL compared to Vav-cre ${ }^{-}$Raptor ${ }^{w t / f l}$ and Vavcre $^{-l+}$ Raptor $^{w t / w t} \mathrm{FL}$ controls $(\mathrm{n}=6)(\mathrm{A})$ and in BM of Mx1-Raptor control (blue) compared to Mx1-Raptor $\mathrm{cKO}(\mathrm{red})$ mice $(\mathrm{n}=6)(\mathrm{B})$; (C) Graph showing surface expression of $\mathrm{S} 1^{\mathrm{lo}} \mathrm{c}-\mathrm{Kit}^{\mathrm{hi}}\left(\mathrm{Sca}-1^{\mathrm{lo}} \mathrm{CD} 117^{\mathrm{hi}}\right), \mathrm{CMP}, \mathrm{GMP}$ populations in E15 FL in Vav-cre ${ }^{-}$Raptor ${ }^{w t / f l}$ (light blue) and Vav-Raptor control (blue) and Vav-cre Raptor $^{\text {wt/ }}$ $f l$ (light red) and Vav-Raptor KO (dark red) $(\mathrm{n}=6)$; (D) Surface expression of megakaryocyte erythrocyte progenitor (MEP) in E13 and E15 FL in Vav-KO mice compared to cre ${ }^{+}$Raptor ${ }^{w t / f l}$ and $V a v$-control (E13 n $\geq 3$; $\mathrm{E} 15 \mathrm{n} \geq 6$ ); (E) BM of $M x 1$-control or $M x 1$-cKO mice showing surface expression of $\mathrm{S}^{\mathrm{lo}} \mathrm{c}-\mathrm{Kit}{ }^{\mathrm{hi}}$, CMP, GMP and MEP populations $(n \geq 5)$; Gene expression of E17 FL demonstrating the fold change in $P u$. 1, early B cell factor 1 (Ebf1), and Pax5 gene expression (F), along with fold changes in gene expression of E15 FL Cebp $\alpha$, Gata1, Gata2, $\beta$ Globin (G), and Kruppel-like factor 1 and 2 (Klf1 and Klf2) (H) in CD45 ${ }^{+}$sorted Vav-Raptor KO FL compared to Vav-Raptor control FL cells $(\mathrm{n} \geq 4)$. All Mxl-cre mice were given 4 doses of poly(I:C) and assessed 5 wk post inoculation. Data are expressed as mean \pm SEM, one way ANOVA ( $p * \leq 0.05 ; * * \leq 0.001$; $* * * \leq 0.0001 ; * * * * \leq 0.00001)$.

FL isolated from Vav-Raptor KO mice revealed no significant changes in the Sca- $1{ }^{\text {lo }} \mathrm{CD} 117^{\text {hi }}$, CMP and GMP populations compared to the control mice (Fig. 3C; Suppl. Fig. 3A). An elevation in the percentage of MEPs in E13 and E15 FLs isolated from Vav-Raptor KO mice suggested a developmental arrest in erythropoiesis at the MEP stage in the absence of Raptor expression at the HSC stage in vivo (Fig. 3D). Interestingly, adult mice with an induced Raptor-deficiency exhibited a significant decrease in Sca- $1^{\mathrm{lo}} \mathrm{CD} 117^{\mathrm{hi}}$ population and MEP population suggesting a block in erythropoiesis prior to the MEP stage in Mx1-Raptor cKO BM (Fig. 3E). Additionally, a significant decrease in GMP population was noted, suggesting that differentiation of CMPs towards GMP and MEPs is deregulated in the BM in Mx1-Raptor cre $\mathrm{CKO}$ mice. The difference in progenitor populations between the FL and BM with Raptor-deficiency highlights the potential for $\mathrm{mTORC1}$ to play similar but not identical roles in lineage maintenance at different stages of development and ontogeny.

In support of our phenotypic analyses showing a block in B cell and RBC development, analysis of key genes responsible for enabling differential lineage commitment revealed a significant downregulation in the expression of B cell specific TFs Ebf1 and Pax5 in FLs isolated from Vav-Raptor KO mice, while Cebp $\alpha$ levels were unaltered (Fig. 3F,G). Assessing erythroid lineage commitment factors, Pu.1, $\beta$-Globin and Gata1 levels were significantly 
A
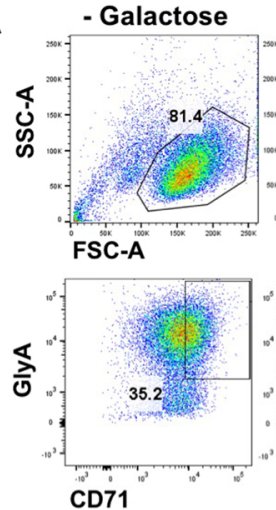
CD71

C

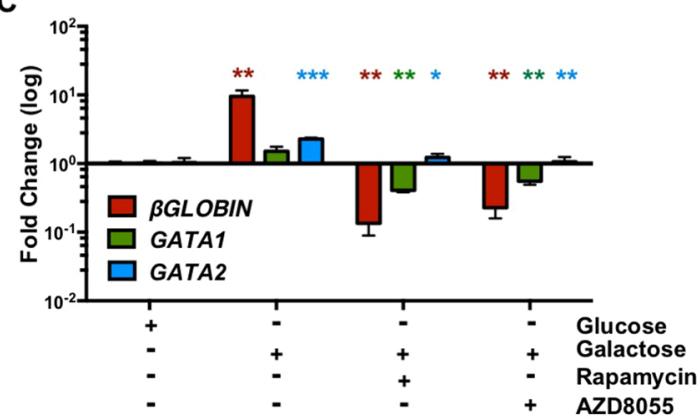

B
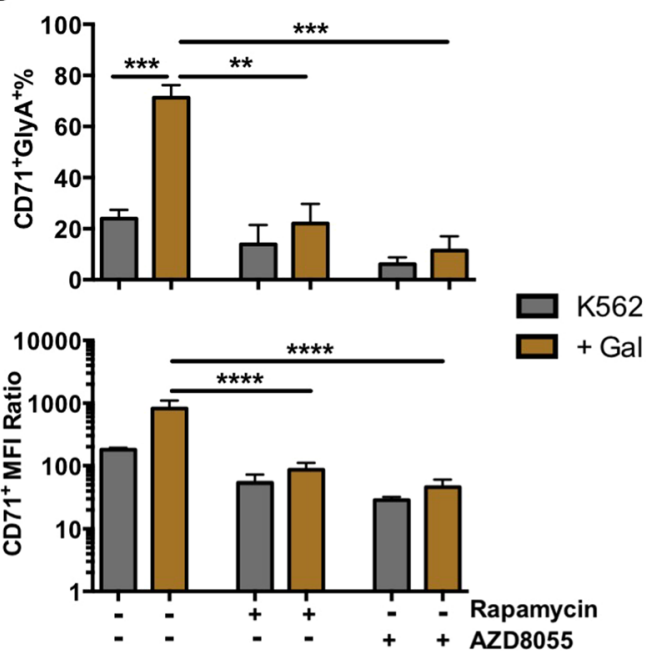

Figure 4. Role of mTORC1 in the differentiation of RBCs in a human cell model at the phenotype and gene transcription level in vitro. (A) Flow cytometry representative plot showing the levels of granularity (FSC-A vs. SSC-A) and in $\mathrm{CD} 71^{+} \mathrm{GlyA}^{+}$surface expression in $\mathrm{K} 562$ cells cultured in complete media or galactosecontaining media. (B) Average percentage of $\mathrm{CD} 1^{+} \mathrm{GlyA}^{+}$cells and MFI ratio of $\mathrm{CD} 71^{+}$expression in $\mathrm{K} 562$ cells $(\mathrm{n}=4)$ when glucose is replaced with galactose (golden bars) in K562 complete media (grey bars). (C) Gene expression of $\beta$-GLOBIN, GATA1 and GATA2 in K562 cells in complete media compared with cells with galactose treatment with or without mTOR inhibitors - rapamycin or AZD8055. Data $(\mathrm{n}=4)$ are expressed as mean \pm SEM, t-test (unpaired) $(\mathrm{p} * \leq 0.05 ; * * \leq 0.001 ; * * \leq 0.0001 ; * * * \leq 0.00001)$.

downregulated in Vav-Raptor KO mice with trends in an increase in Gata2 levels. Additionally, there was a significant downregulation in the expression of Klf1 and Klf2, TFs which play a role in embryonic erythropoiesis and development (Fig. 3H), indicating an aberration in erythropoiesis with Raptor deficiency in Vav-KO FLs.

Inhibition of mTORC1 blocks RBC differentiation in vitro. To further assess the role of mTORC1 in $\mathrm{RBC}$ differentiation in vitro, we made use of the human $\mathrm{BCR}-\mathrm{ABL}^{+}$erythroleukaemia cell line $\mathrm{K} 562$, that differentiates towards a RBC-like lineage when exposed to stress ${ }^{23-25}$. Stress was induced by replacing glucose in complete media with galactose (Gal-media) ${ }^{26}$. A significant increase in the percentage of $\mathrm{CD}^{+} \mathrm{1}^{+} \mathrm{GlyA} \mathrm{A}^{+}$cells, an elevation of the erythroid marker CD71 surface expression and a reduction in cellular granularity was observed (Fig. 4A,B, Suppl. Fig. 4A) in K562 cells cultured in Gal-media, coupled with a significant increase in gene expression of 3-GLOBIN, GATA1 and GATA2 (Fig. 4C). Treatment of K562 cells with either rapamycin (allosteric mTORC1 inhibitor) or AZD8055 (dual mTORC1/2 inhibitor) reduced erythroid differentiation in vitro, as indicated by a significant decrease in the generation of erythroid cells and a reduction in gene expression of erythroid markers, (Fig. 4B,C), indicating mTORC1 inhibition blocks RBC differentiation in vitro.

To assess erythroid colony formation capacity of HPCs in the absence of mTORC1 activity, HPCs were isolated from BM of Mx1-Raptor control or cKO mice and CFC assays were performed. Mx1-Raptor cKO mice lacked CFC activity, as indicated by the lack of colony-forming unit-erythroid (CFU-E), blast-forming unit-erythroid (BFU-E) or granulocyte-erythroid-megakaryocyte-macrophage (GEMM) colonies in the absence of Raptor expression, compared to $\mathrm{cre}^{-}$controls (Fig. 5A,B). Interestingly, myeloid progenitor $\mathrm{CFC}$ assays performed in Vav-cre ${ }^{-}$(Vav-Rictor control) and cre $^{+}$Rictor $^{\text {flffl }}$ (Vav-Rictor KO) HPCs to assess the role of mTORC2 in early myeloid/erythroid colony formation demonstrated a significant increase in CFU-E colonies and a trend in increase in GEMM colonies in Vav-Rictor KO HPCs (Fig. 5C,D), suggesting a suppressive role of mTORC2 in erythropoiesis. Supporting these data, flow cytometric analysis of the CFCs generated showed a decrease in percentage of Ter1 $19^{+}$cells in Mx1-Raptor cKO HPCs, while an increase was noted in Vav-Rictor KO HPCs (Suppl. Fig. 5).

\section{Discussion}

Our data support previously published work demonstrating a critical role of mTORC1 during B cell and erythroid development in two mouse models of Raptor-deletion in vivo, and further demonstrate the importance of mTORC1 function in in vitro RBC differentiation models: CFC assays and human cell line K562. 
A

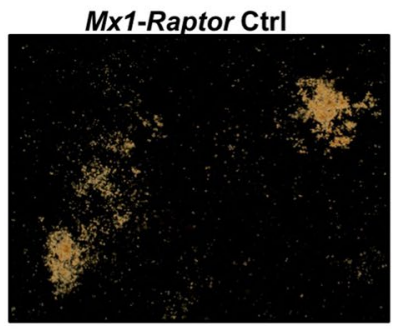

C

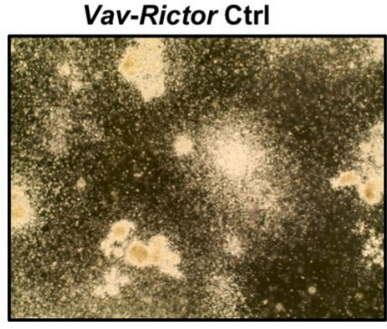

Mx1-Raptor cKO

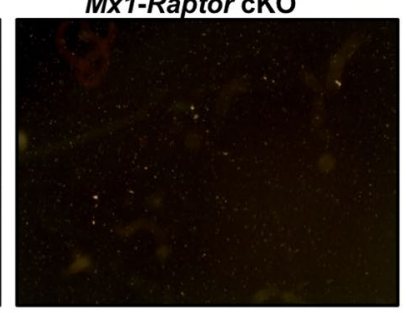

Vav-cre ${ }^{+}$Rictor $\mathrm{KO}$

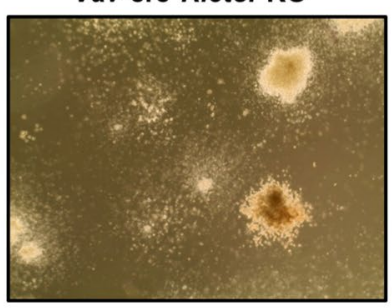

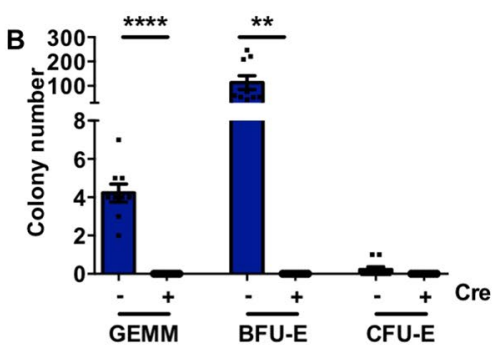

D

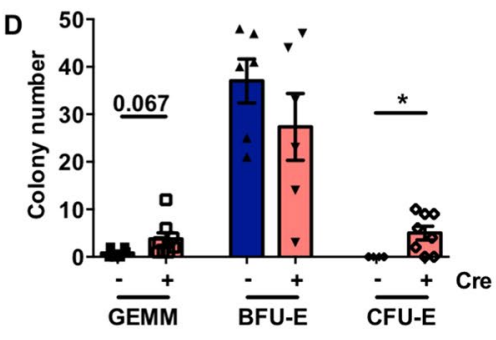

$\mathbf{E}$

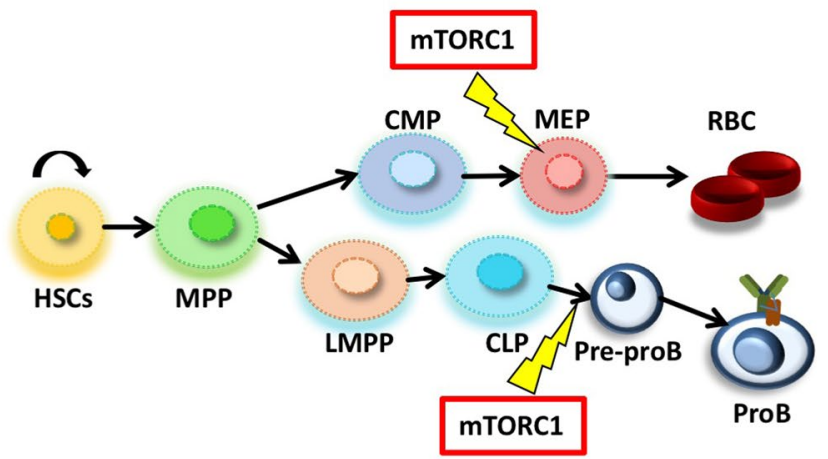

Figure 5. Mx1-Raptor $\mathrm{cKO}$ mice exhibit a functional block in RBC development. (A) Colony formation cell assays (CFCs) which optimise for the growth of erythroid cells were carried out on Mx1-Raptor control (control: left) and Mx1-Raptor cKO (cKO: right) BM enriched for haemopoietic stem/progenitor cells (HSPCs). All Mx1-cre mice were given 4 doses of poly(I:C) assessed 5 wk post last inoculation. (B) Colony counts of different erythroid colonies: colony-forming unit-erythroid (CFU-E), burst-forming unit-erythroid (BFU-E) and CFU of granulocyte-erythroid-macrophage-megakaryocyte (CFU-GEMM) in $\mathrm{cre}^{+}$Raptor ${ }^{\text {fl/fl }} \mathrm{KO}$ models and in $\mathrm{cre}^{-}$controls $(\mathrm{n} \geq 6)$. (C) Colony formation cell assays (CFCs) which optimise for the growth of haematopoietic progenitor colonies were carried out on Vav-Rictor control (control: left) and Vav-Rictor KO (KO: right) BM enriched for HPCs. (D) Colony counts of different haematopoietic colonies: CFU-E, BFU-E and CFU-GEMM in cre $^{+}$Rictor $^{f l / f l} \mathrm{KO}$ models and in cre ${ }^{-}$controls $(\mathrm{n} \geq 6)$. Data are expressed as mean \pm SEM, unpaired t-test $(\mathrm{p} * \leq 0.05 ; * \leq 0.001 ; * * * \leq 0.0001 ; * * * * \leq 0.00001)$. (E) Schematic summary of the role of mTORC1 in B and erythrocyte development. Haemopoietic stem cells (HSCs), which have self-renewal capacity, give rise to multipotent progenitors (MPPs) which can develop towards common myeloid progenitors (CMPs), or lymphoid primed multipotent progenitors (LMPPs), which give rise to common lymphoid progenitors(CLPs) or certain myeloid cell lineages. CMPs further give rise to megakaryocyte-erythroid progenitors (MEPs) which develop into erythrocytes along with other lineages. CLPs give rise to lymphoid lineages including B cell lineage. Yellow lighting arrows represent stages of haemopoietic development where the role of mTORC1 is vital for further development.

Examining the role of mTORC1 in normal erythropoiesis at the HSC stage, in both fetal and adult mice, revealed subtle differences at the two developmental stages. Raptor-deficient embryos were paler than Raptor-control mice, characteristic of mice with RBC deficiency as noted in EPO/EPO-R mice ${ }^{27}$. Knight et al., demonstrated high mTORC1 activity in proE cells (reticulocytes) characterised by elevated phosphorylation of the downstream target $\mathrm{S} 6$ thereby identifying a critical role for mTORC1 in RBC development. Additionally, they showed a link between iron availability and haemoglobin $(\mathrm{Hb})$ synthesis in RBC development with mTORC1 signalling, as RBCs have decreased mTORC1 signalling during iron deficiency (ID) in vitro and in vivo ${ }^{19}$. We observed a significant decrease in Ter $119^{+}$erythrocytes, with an arrest at the MEP stage shown by a significant increase in the MEP population at E15, together with an increase in Sca- $1^{\text {lo }} \mathrm{CD} 117^{\text {hi }}$ cells in the FL of Vav-Raptor KO. This is consistent with published data demonstrating Vav-Raptor KO FL exhibit microcytic anaemia with 
decreased $\mathrm{Hb}$, exhibiting increased proE cells and decreased EryC populations. Conversely, constitutive activation of mTORC1 ( $\mathrm{Vav}$-TSC1 KO) leads to macrocytic anaemia with larger reticulocytes and RBCs with elevated $\mathrm{Hb}$ levels. Despite increased Hb levels in RBCs, there was a decrease in RBC output in the BM (decreased EryB), leading to stress erythropoiesis in the spleen (elevated EryA population) with an overall decrease in $\mathrm{Hb}^{19}$. A steady decline in progenitor populations occurred over time, with no significant difference in MEP populations between the controls and Vav-Raptor KO FL at E17 (data not shown), diminishing any significant differences observed in FL at E13/E15. This is likely due to a transition in haemopoiesis from the FL to the BM, which begins before birth at E16.5 ${ }^{28}$. The block in development of the erythroid lineage was supported by a significant downregulation in Gata1, $\beta$-Globin, Klf1 and Klf2 expression with Raptor-deficiency at the HSC stage, suggesting mTORC1-mediated signalling may aid in the regulation of GATA switching to enable erythropoiesis.

In contrast, induced Raptor-deficiency in adult mice (Mx1-Raptor $\mathrm{CKO})$ is not lethal, and these mice displayed a significant decrease in Sca- $1^{\mathrm{lo}} \mathrm{CD} 117^{\mathrm{hi}}$ and MEP populations in the BM. This may be due to the lineage potential of progenitors differing between fetal and adult mice, as FL HSCs possess a higher proliferative and metabolic capacity (increased oxidative phosphorylation) than BM-derived HSCs which could affect Sca- $1^{\text {lo } C D} 117^{\text {hi }}$ and MEP primitive populations ${ }^{29}$. Nevertheless, there is a trend in decrease in Ter $119^{+}$erythroid population in the $\mathrm{BM}$ of adult mice with Raptor-deficiency, as noted in the Vav-cre model, suggesting a disruption in erythropoiesis in the BM. Indeed, Guo et al., demonstrated that Mx1-Raptor cKO mice have reduced RBCs and $\mathrm{Hb}$ in the BM due to increased apoptosis of RBCs. Additionally, erythrocyte development was blocked at the proE stage $\mathrm{e}^{30} \mathrm{con}^{-}$ firming our results that mTORC1 blocks RBC development. Mx1-Raptor cKO mice exhibited splenomegaly, with the spleen showing a significant increase in Ter $119^{+}$population, which could suggest a possible compensatory mechanism leading to the promotion of erythropoiesis due to a lack of erythrocytes, suggesting extramedullary haemopoiesis in the spleen, as has been shown previously ${ }^{31}$. Alternatively, as the spleen is known to be a main site for erythrophagocytosis, it is possible that the function of these erythrocytes is compromised and are accumulated in the spleen for phagocytosis ${ }^{30}$. We observe splenomegaly and an increase in red pulp. As the red pulp is one of the major sites of erythrocyte destruction, it is possible that an accumulation of erythrocytes in the spleen indicates erythrocyte depletion ${ }^{32}$. The observed differences between the models may be due to the mechanism of Raptor-excision. While both models result in the deletion of Raptor in all haemopoietic lineages, the Mx1-Raptor cKO requires inoculation with poly(I:C), through induction of type 1 interferons, which may disrupt haemopoietic cell survival. Published studies indicate that the impact of poly(I:C) treatment on haemopoietic cells is transient, lasting for up to $48 \mathrm{hr}^{33}$. Here, phenotypic analyses were carried out $5 \mathrm{wk}$ after the last poly(I:C) inoculation, by which time the key poly(I:C) induced effect should be Raptor-excision. While this timeline may enable the outgrowth of undeleted alleles in Mx1-Raptor cKO mice, the optimisation of poly(I:C) induced Raptor-excision leading up to this study reduced the likelihood of this occurring in the timeframe of these experiments, and Raptor expression was consistently downregulated up to $8 \mathrm{wk}$ post-poly(I:C) inoculation.

Using established mTOR inhibitors to block RBC differentiation in the K562 cell line provided support for the results presented from our mouse models, indicating that erythroid development is dependent on mTORC1 activity. Interestingly, a recent report shows that K562 cells express a distinct mTOR-containing complex, mTORC3 (mTOR associated with ETV7) that lacks other mTORC1/2 containing proteins, and is associated with rapamycin-resistance when upregulated in human cancer cells ${ }^{34}$. Our finding that K562 differentiation is inhibited by rapamycin treatment suggests that mTORC3 does not play a major role in this process. Supporting this, Ohyashiki et al. have previously shown that iron regulates mTORC1 signalling in RBCs as iron chelators greatly reduced mTORC1 signalling in K562 cells ${ }^{35}$. Overexpression of eIF4E and sustained mTORC1 expression in erythroid progenitor cell line I/11 also improves differentiation ${ }^{36,37}$. An additional role of mTORC1 in initiation of translation at early stages in RBC development has been reported, demonstrating the regulation of mitochondrial biogenesis from HSPCs to proerythroblasts by $\mathrm{mTORC}^{38}$. This study shows a unique requirement for $\mathrm{mTORC1}$ in RBC development. These data, together with our findings suggest that mTORC1, iron metabolism and erythropoiesis have an inter-dependent regulatory link.

Assessment of erythroid colony formation capacity revealed that Raptor-deficient BM lacked colony formation capacity. Consistent with this, Mx1-Raptor $\mathrm{CKO}$ cells have previously been shown to lack the ability to form CFU-E and BFU-E colonies ${ }^{30}$. Indeed, shRNA targeting S6K1 in murine BM cells significantly decreased CFU-E and BFU-E colony formation capacity demonstrating the importance of mTORC1-S6K axis is mediating erythropoiesis ${ }^{39}$. ER-Raptor cKO LSK cells also lack colony formation capacity which is rescued by the retroviral transduction of Raptor suggesting the importance of mTORC1 in haemopoiesis ${ }^{40}$. Our assessment of Rictor-deficient BM colony formation capacity revealed that Vav-Rictor KO BM formed a significantly higher number of erythroid colonies than their controls. This suggests a possible feedback system for erythroid regulation wherein mTORC1 drives erythroid formation, whereas mTORC2 suppresses erythropoiesis. Our study compared the Mx1-Raptor $\mathrm{CKO}$ and Vav-Rictor KO models. Vav-mediated KO models generate a constitutive KO, which can result in signalling pathway adaptation ${ }^{41}$. Indeed, crosstalk between ERK-MAPK and mTOR signalling pathways has recently been demonstrated, with ERK activity regulating $\mathrm{mTORC} 1$ activation to limit the promotion of HSC cycling in favour of quiescence. HSCs derived from MEK1-cKO mice exhibit exhaustion due to increased mTORC1-mediated ROS production resulting in increased mitochondrial damage ${ }^{42}$. While each model has a different timing for gene deletion, the cells placed in CFC assays were removed from Mx1-Raptor mice 5 wk after inoculation with poly(I:C) in vivo, thus inducing optimal Raptor excision.

Of note, publications also suggest that mTORC1 negatively regulates erythropoiesis, via heme-regulated eIF $2 \alpha$ kinase (HRI) and activation transcription factor 4 (ATF4), in a mouse model of ID. HRI is activated in response to heme deficiency, thereby stimulating ATF4 to regulate $\mathrm{Hb}$ levels via the translation of stress response genes. In mutant mice that lack phosphorylated eIF $2 \alpha(\mathrm{eIF} 2 \alpha \mathrm{P})$ in the RBC lineage $(e A A)^{43}$, mTORC1 activity is elevated in erythroid precursors. This study demonstrated that eIF $2 \alpha \mathrm{P}$ and ATF4 of HRI-integrated stress response are required in ID to repress mTORC1 signalling and mitigate ineffective erythropoiesis. As such, pharmacological 
inhibition of mTORC1 significantly improved RBC production and differentiation, and increased Hb levels in the blood ${ }^{43}$. Furthermore, constitutive activation of mTORC1 in haemopoietic lineages exhibited macrocytic hyperchromic anaemia with splenomegaly and ineffective erythropoiesis ${ }^{19}$, similar to that seen in $\mathrm{HRI}^{-1-}$ mice. However, although ATF4 is required to inhibit mTORC1 signalling, ATF4 ${ }^{-1-}$ mice develop microcytic hypochromic anaemia, unlike $\mathrm{HRI}^{-1-}$ mice ${ }^{43}$. These studies suggest that mTORC1 activity plays an inhibitory role in erythropoiesis during ID anaemia. Furthermore, Foxo $3^{-1-}$ mice (model with similar genotypic profile to $\beta$-thalassemia), exhibit increased phosphorylation of mTOR targets including AKT, S6, 4EBP1, suggesting aberrant mTOR signalling in diseased conditions. Treatment of Foxo $3^{-1-}$ mice with rapamycin increased RBC counts and $\mathrm{Hb}$ levels, with increased cell cycling in immature erythrocytes compared to untreated mice ${ }^{39}$. Therefore, in contrast to normal erythropoiesis, where $\mathrm{mTORC1}$ plays a fundamental role in RBC generation, diseased conditions alter mTORC1 signalling to disrupt erythropoiesis, which is overcome by mTORC1 inhibitors.

The early block in B cell lineage commitment and development in the FL and BM of both Raptor-deficient models, mirrored that described previously in Mx1-Raptor $\mathrm{cKO}$ mice $^{31}$. The observed increase in LSK populations, which normally give rise to pre-proB cells, in both BM and E15 FL suggests a block at the LSK stage during B cell development with Raptor-deficiency at the fetal and in adult stages of development. These findings were supported by the observed downregulation in Ebf1 and Pax5, TFs essential for B cell lineage commitment. A similar trend has been observed in the BM of $m T O R-c K O$ mice, and the spleen of Mx1-Raptor cKO mice, with the majority of LSK cells in S phase suggesting increased cell cycling due to perturbations in mTORC1 signalling ${ }^{29,30}$. Iwata et al., demonstrate a block in B cell development at the pre-B cell stage within Mb1-Raptor cKO adult mice, which is not rescued by the introduction of an anti-apoptotic $B c l_{X L}$ transgene suggesting that this block, caused by the lack of mTORC1, is independent of $\mathrm{BCL}_{\mathrm{XL}}{ }^{44}$. Our model, which excises Raptor at the HSC stage, demonstrates a requirement for mTORC1 signalling for B cell lineage commitment. In ER-Raptor cKO BM there is an increase in LSK cells together with a decrease in phosphorylation of 4EBP1 and S6 and decrease in mature $\mathrm{B}$ cells ${ }^{40}$ indicating that the role of $\mathrm{mTORC} 1$ in B lymphopoiesis is not model specific. Indeed, Zeng et al. highlight the importance of mTORC1 specifically in B cell development and proliferation, but not survival, in an IL7R-mTORC1-Myc dependent and STAT5-independent manner, as the loss of mTORC1 leads to a block at the proB cell stage in CD2-mTOR and CD2-Raptor cKO models ${ }^{45}$. Furthermore, Keating et al. demonstrated a critical role for mTORC1 in B cell class switching and anti-viral responses, with Rosa26-Raptor KO mice having reduced germinal centre formation in response to influenza infection, a finding that was replicated with rapamycin treatment. Interestingly, rapamycin treatment prevented B cell class-switching, yielding antibodies that mediated heterosubtypic protection, by targeting more conserved regions of hemagglutinin, a surface protein expressed on viruses $^{46}$. H\&E staining of spleens isolated from $M x 1$-Raptor $\mathrm{CKO}$ mice lack a defined architecture and displayed a reduction in marginal zones and germinal centres (data not shown).

In agreement with previously published data, similar roles of mTORC1 in myeloid lineage development were observed in both fetal and adult mice with a significant increase in $\mathrm{CD} 11 \mathrm{~b}^{+} \mathrm{Gr} 1^{-}$populations and a concomitant decrease in $\mathrm{CD}_{11 \mathrm{~b}^{+}} \mathrm{Gr1}^{+}$populations in both Vav-Raptor KO FL and Mx1-Raptor CKO BM ${ }^{30,31}$. This result has been recapitulated in $E R$-cre model suggesting that the role of mTORC1 in myeloid development is not model specific ${ }^{40}$. We observed a decrease in GMP population leading to a decrease in mature myeloid population with Raptor-deficiency in Mx1-Raptor cKO mice. Indeed, a decrease in CMPs has previously been reported in $M x 1$-Raptor $\mathrm{CKO}$ mice $^{30}$ supporting the decrease in mature myeloid lineage with Raptor-deficiency. However, the increase in $\mathrm{CD}_{11} \mathrm{~b}^{+}$population in both models also suggests that even though there is a block in early myeloid populations, it does not disrupt the ability of the progenitors to develop into immature myeloid cells. ER-Raptor

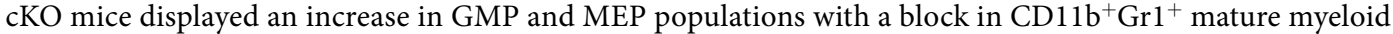
populations with no changes in pS6, alluding to the phenotypic differences between KO models. The lack of change in pS6 expression in myeloid cells (with a significant decrease in pS6 in B cells) suggests a differential regulation of mTORC1 activity in different haemopoietic lineages ${ }^{40}$. Nonetheless, mTORC1 plays a role in the maturation of myeloid cells in both adult and fetal models. While previous studies support a cell autonomous effect of Raptor-deletion in the development of haemopoietic lineages ${ }^{30,31}$, mixed BM chimeras should be performed to confirm our results, as myeloid lineage expansion has the potential to inhibit erythroid and lymphoid lineages ${ }^{47,48}$. In addition, deletion of Raptor in HSCs has been shown to affect osteoclast generation, which in turn would impact the BM microenvironment and subsequent haemopoiesis ${ }^{49}$.

Taken together, we performed analyses in two different mTORC1 KO mouse models at different stages of development in vivo and two distinct RBC differentiation assays in vitro to tease out the role of mTORC1 in haemopoietic lineage development. We confirm the fundamental role of mTORC1 in haemopoiesis, during RBC and $\mathrm{B}$ cell lineage commitment both in fetal and adult mice (Fig. 5E).

\section{Materials and Methods}

Mice. Mice expressing floxed Raptor (Raptor $\left.{ }^{f l / f l}\right)$ or Rictor (Rictor $\left.{ }^{f l / f l}\right)$ constructs were obtained from Prof. Michael N. Hall (University of Basel, Switzerland) ${ }^{50}$. Prof. Tessa L. Holyoake (University of Glasgow, UK) provided transgenic mice expressing $M \times 1$-cre and $V a v$-cre, which were bred and maintained at the Beatson Research Unit (Glasgow, UK). All experimental protocols were approved by the local AWERB committee and national home office, and all methods were carried out in accordance with standard animal housing conditions under local and home office regulations. Mxl-cre ${ }^{-1+}$ or Vav-cre ${ }^{-1+}$ transgenic mice were crossed with Raptor ${ }^{f l f l}$ or Rictor rlffl $^{f l}$ to obtain the desired KO models. The $M x 1$ promoter was activated in $M x 1-$ cre $^{-l+}$-Raptor ${ }^{\mathrm{fl} / \mathrm{fl}}$ or -Rictor ${ }^{f l / f l}$ mice by inoculating the mice with 4 doses of $10 \mathrm{mg} / \mathrm{kg}$ polyionosinic:polycytidylic acid (poly(I:C); TLR3 agonist) once every 2 days to induce a conditional $\mathrm{KO}(\mathrm{cKO})$. The mice were assessed $5 \mathrm{wk}$ after the last inoculation. No significant differences were observed in organ cellularity, and $\mathrm{B} /$ myeloid lineage percentage and number when B6.SJL wild type mice and $M x 1$-Cre ${ }^{-}$Raptor ${ }^{\ell / f l}$ mice were compared after treatment with poly(I:C); Supplemental Fig. 6). The Vav promoter is active at E13 in HSCs, thereby inducing a haemopoietic-restricted KO. Haemopoietic 
lineage cells in a single cell suspension were isolated from BM, spleen and blood of transgenic mice, and filtered through a $70 \mu \mathrm{m}$ nylon mesh (Fisher Scientific, Leicestershire, UK). The cells collected from the BM and spleen were enriched for haemopoietic lineage cells by density centrifugation using Lympholyte-Mammal (Cedarlane, Canada), centrifuging the cell suspension for $20 \mathrm{~min}$ at $625 \mathrm{~g}$ at RT. Thereafter the cells were washed in PBS, centrifuged at $500 \mathrm{~g}$ for $10 \mathrm{~min}$ at RT and counted.

Flow cytometry. Samples derived from the organs were prepared for flow cytometry as described previously ${ }^{51}$. All antibodies were purchased from BD Biosciences (Oxford, UK), except Gr-1 (Clone RB6-8C5; eBiosciences) and Ter119 (Clone TER-119; BioLegend). K562 cells with/without treatment were harvested and $2 \times 10^{5}$ cells were stained with GlyA (Clone GAR2) and CD71 (Clone AC102) from Miltenyi Biotec. All samples were suspended in $100 \mu \mathrm{l}$ PBS and acquired using the FACSDiva software package (BD Biosciences) on the FACSCantoII flow cytometer. The resultant data were analysed using FlowJo software (Tree Star Inc., OR).

qRT-PCR. RNA was extracted from fresh cells by following the RNAeasy Qiagen Kit protocol. cDNA was then made from the RNA using standard protocols provided by Invitrogen. Alternatively, $\mathrm{CD} 45^{+}$cells were sorted into the PCR tubes ( 300 cells/tube) containing the one-step PCR master mix of $5 \mu$ l (Cells direct $2 \mathrm{x}$ reaction $(2.8 \mu \mathrm{l}), 0.2 \mathrm{x}$ Primer mix $(1.4 \mu \mathrm{l})$, RNAase out $(0.056 \mu \mathrm{l})$, Superscript III RT/Platinum Taq Mix $(0.112 \mu \mathrm{l})$, TE Buffer $(0.672 \mu \mathrm{l})$ (part of SuperScript ${ }^{\mathrm{TM}}$ III Platinum ${ }^{\mathrm{TM}}$ One-Step qRT-PCR Kit, Invitrogen)). Samples were run on a PCR with the following conditions: $50^{\circ} \mathrm{C}$ for $15 \mathrm{~min}, 95^{\circ} \mathrm{C}$ for $2 \mathrm{~min}$ followed by 20 cycles of $95^{\circ} \mathrm{C}$ for $15 \mathrm{sec}$ and $65^{\circ} \mathrm{C}$ for $4 \mathrm{~min}$. The samples were then held at $4^{\circ} \mathrm{C}$. After the completion of the PCR, the samples were diluted $10 \mathrm{x}$ with TE buffer $(45 \mu \mathrm{l})$. qPCR was carried out by using $300 \mathrm{nM}$ of forward and reverse primer for each gene. All reactions were performed in technical triplicates and at least three biological replicates using the 7900HT Fast Real-Time PCR system (Applied Biosystems, Warrington, UK), programmed to complete 40 cycles as follows: $50^{\circ} \mathrm{C}$ for $20 \mathrm{~min}, 95^{\circ} \mathrm{C}$ for $10 \mathrm{~min}$, followed by $40 \mathrm{cycles}$ of $95^{\circ} \mathrm{C}$ for $15 \mathrm{sec}$, and $60^{\circ} \mathrm{C}$ for $1 \mathrm{~min}$. The primers used are listed in Supplementary Table 1. The q/RT-PCR follows the MIQE guidelines ${ }^{52}$.

Erythroid differentiation of K562 cells. For normal cell growth, K562 cells were cultured in RPMI1640 supplemented with $10 \% \mathrm{FBS}, 50 \mathrm{U} / \mathrm{ml}$ penicillin, $50 \mu \mathrm{g} / \mathrm{ml}$ streptomycin and $2 \mathrm{mM}$ L-Glutamine (complete media). To induce erythroid differentiation, K562 cells were cultured in RPMI-1640 (no glucose) supplemented with $10 \% \mathrm{FBS}, 50 \mathrm{U} / \mathrm{ml}$ penicillin, $50 \mu \mathrm{g} / \mathrm{ml}$ streptomycin, $2 \mathrm{mM} \mathrm{L}$-Glutamine and $11 \mathrm{mM}$ Galactose (Gal-media). For erythroid differentiation, K562 cells $\left(2 \times 10^{5}\right)$ were cultured in either complete media or Gal-media for up to 7 days and then analysed by flow cytometry or qPCR. For drug treatments, cells were treated with $10 \mathrm{nM}$ rapamycin (allosteric mTORC1 inhibitor) or $100 \mathrm{nM}$ AZD8055 (competitive dual mTORC1/2 inhibitor) or vehicle control (DMSO) as indicated.

Colony forming cell (CFC) assay. Bones from transgenic mice were crushed and enriched for haemopoietic lineages as described above. Haemopoietic progenitor cells (HPCs) were isolated using the MACS mouse CD117 MicroBeads according to the manufacturer's protocol (Miltenyi Biotec, Surrey, UK). Up to $2 \times 10^{4}$ cells were then plated in MethoCult ${ }^{\mathrm{TM}} \mathrm{M} 3334$ erythroid colony promoting methylcellulose or MethoCult ${ }^{\mathrm{TM}} \mathrm{GF}$ $\mathrm{M} 3434$, in $6 \mathrm{~cm}$ dishes as per the manufacturer's protocol (Stemcell Technologies, Grenoble, France). The dishes were monitored and colonies were counted.

Statistics. The Chi Test was used to assess whether there were statistical differences between the different genotypes generated from crossing $\mathrm{Vav}$-cre ${ }^{-l+}$ Raptor ${ }^{f l / f l}$ mice. Chi Test calculates deviations by chance if the expected ratios are known. Medelian ratios were the expected ratios, which were compared to the genotypes observed at weaning stage of mice. The statistical significance, if any, is calculated using the Chi Test taking into account the degrees of freedom ${ }^{53}$. Statistical analyses were carried out between the data sets using GraphPad Prism 6 Software (San Diego, California, USA). Statistical analyses carried out were unpaired t tests, or for data with multiple comparisons one way ANOVA, where $\mathrm{p} \leq 0.05$ is considered significant. $* \leq 0.05$; $* * 0.001$; $* * * \leq 0.0001 ; * * * * \leq 0.00001$

\section{Data availability}

No datasets were generated or analysed during the current study.

Received: 13 May 2019; Accepted: 26 October 2019;

Published online: 15 November 2019

\section{References}

1. Notta, F. et al. Distinct routes of lineage development reshape the human blood hierarchy across ontogeny. Science 351, aab2116 (2016).

2. Woolthuis, C. M. \& Park, C. Y. Hematopoietic stem/progenitor cell commitment to the megakaryocyte lineage. Blood 127, $1242-1248$ (2016).

3. Valent, P. et al. Normal and pathological erythropoiesis in adults: from gene regulation to targeted treatment concepts. Haematologica 103, 1593-1603 (2018).

4. Fujiwara, Y., Browne, C. P., Cunniff, K., Goff, S. C. \& Orkin, S. H. Arrested development of embryonic red cell precursors in mouse embryos lacking transcription factor GATA-1. Proc. Natl. Acad. Sci. USA 93, 12355-12358 (1996).

5. Dijon, M. et al. The role of Ikaros in human erythroid differentiation. Blood 111, 1138-1146 (2008).

6. Kueh, H. Y., Champhekhar, A., Nutt, S. L., Elowitz, M. B. \& Rothenberg, E. V. Positive feedback between PU.1 and the cell cycle controls myeloid differentiation. Science 341, 670-673 (2013).

7. Nerlov, C. \& Graf, T. PU.1 induces myeloid lineage commitment in multipotent hematopoietic progenitors. Genes Dev. 12, 2403-2412 (1998). 
8. Suh, H. C. et al. C/EBP $\alpha$ determines hematopoietic cell fate in multipotential progenitor cells by inhibiting erythroid differentiation and inducing myeloid differentiation. Blood 107, 4308-4316 (2006).

9. Zhang, P. et al. Negative cross-talk between hematopoietic regulators: GATA proteins repress PU.1. Proc. Natl. Acad. Sci. USA 96, 8705-8710 (1999).

10. Zhang, P. et al. PU.1 inhibits GATA-1 function and erythroid differentiation by blocking GATA-1 DNA binding. Blood 96, 2641-2648 (2000).

11. Anderson, K. P., Crable, S. C. \& Lingrel, J. B. The GATA-E box-GATA motif in the EKLF promoter is required for in vivo expression. Blood 95, 1652-1655 (2000).

12. Siatecka, M. \& Bieker, J. J. The multifunctional role of EKLF/KLF1 during erythropoiesis. Blood 118, 2044-2054 (2011)

13. Asano, H. \& Stamatoyannopoulos, G. Activation of $\beta$-Globin Promoter by Erythroid Krüppel-Like Factor. Mol. Cell. Biol. 18, 102-109 (1998)

14. Novodvorsky, P. \& Chico, T. J. A. The role of the transcription factor KLF2 in vascular development and disease. Prog. Mol. Biol. Transl. Sci. 124, 155-188 (2014).

15. Nutt, S. L. \& Kee, B. L. The transcriptional regulation of B cell lineage commitment. Immunity 26, 715-725 (2007).

16. Hagman, J. \& Lukin, K. Transcription factors drive B cell development. Curr. Opin. Immunol. 18, 127-134 (2006).

17. Vadlakonda, L., Dash, A., Pasupuleti, M., Anil Kumar, K. \& Reddanna, P. The Paradox of Akt-mTOR Interactions. Front. Oncol. 3 (2013).

18. Treins, C., Warne, P. H., Magnuson, M. A., Pende, M. \& Downward, J. Rictor is a novel target of p70 S6 kinase-1. Oncogene 29, 1003-1016 (2009).

19. Knight, Z. A., Schmidt, S. F., Birsoy, K., Tan, K. \& Friedman, J. M. A critical role for mTORC1 in erythropoiesis and anemia. eLife 3 (2014).

20. Diekmann, F. et al. mTOR inhibition and erythropoiesis: microcytosis or anaemia? Nephrol. Dial. Transplant. Off. Publ. Eur. Dial. Transpl. Assoc. - Eur. Ren. Assoc. 27, 537-541 (2012).

21. Wang, J. et al. mTOR Inhibition Improves Anaemia and Reduces Organ Damage in a Murine Model of Sickle Cell Disease. Br. J. Haematol. 174, 461-469 (2016).

22. Mombaerts, P. et al. Mutations in T-cell antigen receptor genes $\alpha$ and $\beta$ block thymocyte development at different stages. Nature 360, 225-231 (1992)

23. Furusawa, M., Onishi, T., Taira, T., Iguchi-Ariga, S. M. \& Ariga, H. AMY-1 is a trigger for the erythrocyte differentiation of K562 cells. Int. J. Oncol. 16, 339-384 (2000).

24. Ida, C., Ogata, S., Okumura, K. \& Taguchi, H. Induction of Differentiation in K562 Cell Line by Nicotinic Acid-Related Compounds. Biosci. Biotechnol. Biochem. 73, 79-84 (2009).

25. Isoda, H. et al. Analysis of the erythroid differentiation effect of flavonoid apigenin on K562 human chronic leukemia cells. Chem. Biol. Interact. 220, 269-277 (2014).

26. Karvela, M. et al. ATG7 regulates energy metabolism, differentiation and survival of Philadelphia-chromosome-positive cells. Autophagy 12, 936-948 (2016).

27. Wu, H., Liu, X., Jaenisch, R. \& Lodish, H. F. Generation of committed erythroid BFU-E and CFU-E progenitors does not require erythropoietin or the erythropoietin receptor. Cell 83, 59-67 (1995).

28. Jagannathan-Bogdan, M. \& Zon, L. I. Hematopoiesis. Dev. Camb. Engl. 140, 2463-2467 (2013).

29. Manesia, J. K. et al. Highly proliferative primitive fetal liver hematopoietic stem cells are fueled by oxidative metabolic pathways. Stem Cell Res. 15, 715-721 (2015).

30. Guo, F. et al. Mouse gene targeting reveals an essential role of mTOR in hematopoietic stem cell engraftment and hematopoiesis. Haematologica 98, 1353-1358 (2013).

31. Kalaitzidis, D. et al. mTOR complex 1 plays critical roles in hematopoiesis and Pten-loss-evoked leukemogenesis. Cell Stem Cell 11, 429-439 (2012).

32. Knutson, M. \& Wessling-Resnick, M. Iron Metabolism in the Reticuloendothelial System. Crit. Rev. Biochem. Mol. Biol. 38, 61-88 (2003).

33. Essers, M. A. et al. IFNalpha activates dormant haematopoietic stem cells in vivo. Nature 458, 904-908 (2009).

34. Harwood, F. C. et al. ETV7 is an essential component of a rapamycin-insensitive mTOR complex in cancer. Sci. Adv. 4, eaar3938 (2018).

35. Ohyashiki, J. H. et al. The oral iron chelator deferasirox represses signaling through the mTOR in myeloid leukemia cells by enhancing expression of REDD1. Cancer Sci. 100, 970-977 (2009).

36. Blázquez-Domingo, M., Grech, G. \& von Lindern, M. Translation Initiation Factor 4E Inhibits Differentiation of Erythroid Progenitors. Mol. Cell. Biol. 25, 8496-8506 (2005).

37. Grech, G. et al. Igbp1 is part of a positive feedback loop in stem cell factor-dependent, selective mRNA translation initiation inhibiting erythroid differentiation. Blood 112, 2750-2760 (2008).

38. Liu, X. et al. Regulation of mitochondrial biogenesis in erythropoiesis by mTORC1-mediated protein translation. Nat. Cell Biol. 19, 626-638 (2017)

39. Zhang, X. et al. FOXO3-mTOR metabolic cooperation in the regulation of erythroid cell maturation and homeostasis. Am. J. Hematol. 89, 954-963 (2014).

40. Hoshii, T. et al. mTORC1 is essential for leukemia propagation but not stem cell self-renewal. J. Clin. Invest. 122, 2114-2129 (2012).

41. Malik, N., Sansom, O. J. \& Michie, A. M. The role of mTOR-mediated signals during haemopoiesis and lineage commitment. Biochem. Soc. Trans. 46, 1313-1324 (2018).

42. Baumgartner, C. et al. An ERK-Dependent Feedback Mechanism Prevents Hematopoietic Stem Cell Exhaustion. Cell Stem Cell 22, 879-892 (2018).

43. Zhang, S. et al. HRI coordinates translation by $\mathrm{eIF} 2 \alpha \mathrm{P}$ and $\mathrm{mTORC} 1$ to mitigate ineffective erythropoiesis in mice during iron deficiency. Blood 131, 450-461 (2018).

44. Iwata, T. N. et al. Conditional Disruption of Raptor Reveals an Essential Role for mTORC1 in B Cell Development, Survival, and Metabolism. J. Immunol. 197, 2250-2260 (2016).

45. Zeng, H. et al. Discrete roles and bifurcation of PTEN signaling and mTORC1-mediated anabolic metabolism underlie IL-7-driven B lymphopoiesis. Sci. Adv. 4 (2018).

46. Keating, R. et al. The kinase mTOR modulates the antibody response to provide cross-protective immunity to lethal infection with influenza virus. Nat. Immunol. 14, 1266-1276 (2013).

47. Suh, H. C. et al. C/EBPalpha determines hematopoietic cell fate in multipotential progenitor cells by inhibiting erythroid differentiation and inducing myeloid differentiation. Blood 107, 4308-4316 (2005).

48. Maeda, K. et al. IL-6 blocks a discrete early step in lymphopoiesis. Blood 106, 879-855 (2005).

49. Huynh, H. D. \& Wan, Y. mTORC1 impedes osteoclast differentiation via calcineurin and NFATc1. Commun. Biol. 1, 29 (2018).

50. Bentzinger, C. F. et al. Skeletal Muscle-Specific Ablation of raptor, but Not of rictor, Causes Metabolic Changes and Results in Muscle Dystrophy. Cell Metab. 8, 411-424 (2008).

51. Nakagawa, R., Soh, J. W. \& Michie, A. M. Subversion of Protein Kinase C $\alpha$ Signaling in Hematopoietic Progenitor Cells Results in the Generation of a B-Cell Chronic Lymphocytic Leukemia-Like Population In vivo. Cancer Res. 66, 527-534 (2006). 
52. Bustin, S. A. et al. The MIQE Guidelines: Minimum Information for Publication of Quantitative Real-Time PCR Experiments. Clin. Chem. 55, 611-622 (2009).

53. Griffiths, A. J., Miller, J. H., Suzuki, D. T., Lewontin, R. C. \& Gelbart, W. M. Chi-square test. in An Introduction to Genetic Analysis. (W. H. Freeman, 2000).

\section{Acknowledgements}

This study was funded by a Bloodwise project grant awarded to AMM, \& OJS (15041). Cell sorting facilities were funded by the Kay Kendall Leukaemia Fund awarded to AMM (KKL501) and the Howat Foundation. JH is funded by the Bloodwise project grant (15041) and NM was funded by a MRC-DTP PhD studentship.

\section{Author contributions}

N.M. designed/performed the majority of experiments, analyzed and interpreted the data, carried out statistical analysis and drafted the manuscript; J.C., J.H. and C.E. performed some experiments, assisted in experimental design and carried out data analysis; K.D. provided technical assistance with the in vivo models and analyzed the data; O.S. obtained funding for the study and supervised the studies; A.M.M. obtained funding for the study, designed the research, supervised the studies, analyzed and interpreted the data and wrote the manuscript. All authors reviewed the manuscript.

\section{Competing interests}

The authors declare no competing interests.

\section{Additional information}

Supplementary information is available for this paper at https://doi.org/10.1038/s41598-019-53141-1.

Correspondence and requests for materials should be addressed to A.M.M.

Reprints and permissions information is available at www.nature.com/reprints.

Publisher's note Springer Nature remains neutral with regard to jurisdictional claims in published maps and institutional affiliations.

(c) Open Access This article is licensed under a Creative Commons Attribution 4.0 International License, which permits use, sharing, adaptation, distribution and reproduction in any medium or format, as long as you give appropriate credit to the original author(s) and the source, provide a link to the Creative Commons license, and indicate if changes were made. The images or other third party material in this article are included in the article's Creative Commons license, unless indicated otherwise in a credit line to the material. If material is not included in the article's Creative Commons license and your intended use is not permitted by statutory regulation or exceeds the permitted use, you will need to obtain permission directly from the copyright holder. To view a copy of this license, visit http://creativecommons.org/licenses/by/4.0/.

(C) The Author(s) 2019 